\title{
Extended IACS incremental-iterative method for calculation of hull girder ultimate strength in analysis and...
}

Article $\cdot$ January 2010

\section{CITATION}

1

3 authors:

\section{Stanislav Kitarovic}

University of Zagreb

17 PUBLICATIONS 27 CITATIONS

SEE PROFILE

Vedran Zanic

39 PUBLICATIONS 144 CITATIONS

SEE PROFILE
READS

36

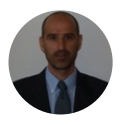

Jerolim Andrić

University of Zagreb

27 PUBLICATIONS 64 CITATIONS

SEE PROFILE

Some of the authors of this publication are also working on these related projects: 


\title{
Extended IACS incremental-iterative method for calculation of hull girder ultimate strength in analysis and design
}

\author{
S. Kitarovic, J. Andric \& V. Zanic \\ University of Zagreb, Faculty of Mechanical Engineering and Naval Architecture, Zagreb, Croatia
}

ABSTRACT: Theoretical background and operational aspects are given for the proposed extension of the IACS incremental-iterative method. Method is used for the assessment of the hull girder ultimate bending moment as one of the design attributes within optimization based decision making process and is implemented in OCTOPUS design environment. Examples of application are given and the general conclusion based on the results obtained is presented.

\section{INTRODUCTION}

Since the ultimate strength might be perceived as the most meaningful safety measure of the ship's hull girder structure, prediction of the ultimate bending moment becomes essential and unavoidable part of the ship structural concept design process. Methods employed should support multiple failure modes and their interactions, while giving precise prediction of collapse and post-collapse behavior of the structural members involved (particularly those under compression). On the other hand, multiple executions within design loop demand utilization of stable, robust and sufficiently fast algorithms.

Consideration of the above stated demands resulted in development of the improved incrementaliterative method for longitudinal ultimate strength assessment based on IACS prescribed incrementaliterative method. Incorporated method particularities include contemporary advances which improve the accuracy during multi-deck ship application, as well as the ability to consider vertical shear force influence on the ultimate hull girder strength.

Purpose of this paper is to give an insight into theoretical background and operational aspects of this method. It is to be used for the assessment of the ultimate bending moment as one of the design attributes within optimization based decision making process, as implemented in OCTOPUS design environment (Zanic et al. 2007).

\section{EXTENSION OF THE BASIC METHOD}

\subsection{General remarks}

Ultimate longitudinal strength is defined herein as the value of bending moment at which the flexural stiffness of the hull girder (i.e. the slope of moment to curvature curve) assumes the value of zero, as illustrated by Figure 1.

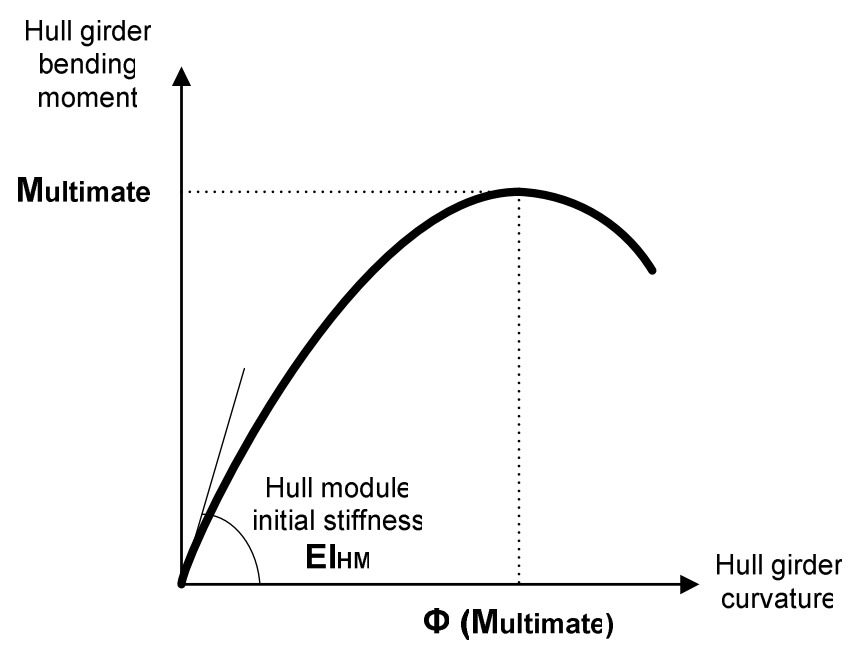

Figure 1. Qualitative hull module moment to curvature (M- $\Phi$ ) response curve, obtained by utilization of the Bernoulli-Euler beam idealization of the hull girder.

The general approach for assessment of the moment to curvature relationship used in IACS prescribed incremental-iterative method is similar to the one originally proposed by well known and widely spread Smith's method (Smith 1977) and will not be further discussed here. Modifications of the basic method are introduced in effort to enable inclusion of the effects disregarded by the basic method and thus improve the overall accuracy of the analysis. Influence of the shear stress and deck efficiency is incorporated into basic method as illustrated by the Figure 2, which represents general flowchart of the proposed method. 


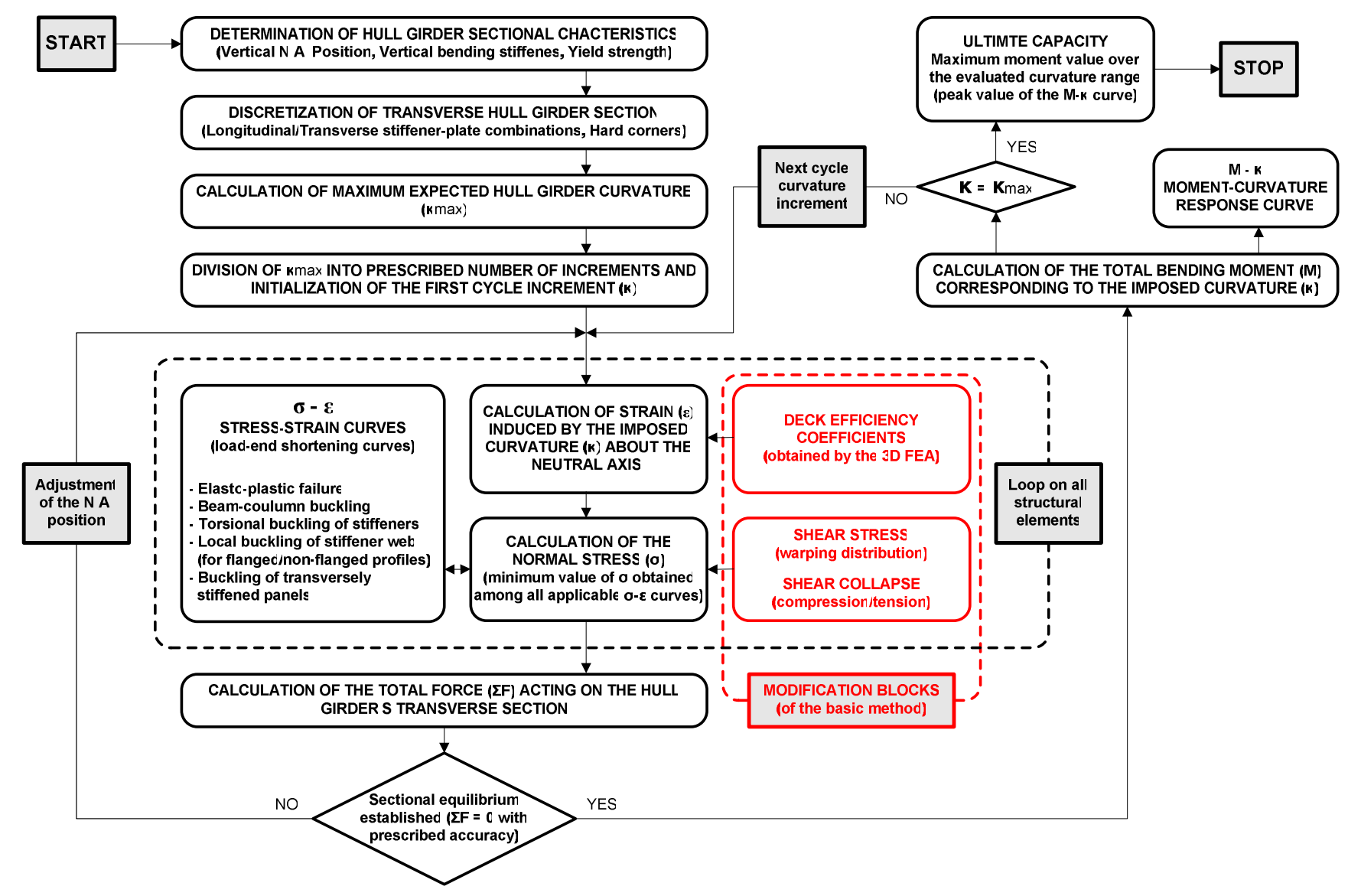

Figure 2. Flowchart of the proposed method.

\subsection{Inclusion of the shear stress influence}

Longitudinal ultimate strength is usually analyzed at the hull girder cross-section with maximum bending moment, where the shear force is negligible. Accounting for shear might be interesting when there is a cross-section along the hull girder with less then maximum value of bending moment, but with significant value of the shear force (cases of alternate loading conditions). Since in this case it is not so obvious whether the hull girder will collapse at the section with the maximum value of the vertical bending moment or at the sections with high vertical bending moment and shear force values, both scenarios deserve the due consideration.

The effect of the vertical shear force on the hull girder ultimate strength is considered trough the influence of the warping induced shear stress distribution of the hull module on the collapse (buckling, yield) of the principal structural members.

For the structural evaluation of primary response at the concept design level the beam idealization is often used. Since this evaluation is based on the extended beam theory which needs cross-sectional characteristics usually obtained using analytical methods, this can be very complicated for the realistic combinations of interconnected open and closed thin-walled (un)symmetric cross sections.
Application of the energy based numerical methods gives an opportunity for alternative approach based on decomposition of a cross section into the line finite elements between nodes $i$ and $j$ (Figure 3) with coordinates $\left(y_{i}, z_{i}\right),\left(y_{j}, z_{j}\right)$; element thickness $t^{e}$; material characteristics (Young's modulus $E$, shear modulus $G$ ) and material efficiency $R N$ and $R S$ (due to hatches, cutouts, lightening holes, etc.) with respect to normal/shear stresses respectively.

The methodology (Zanic \& Prebeg 2005) is based on application of the principle of minimum total potential energy with respect to the parameters which define the displacement fields of the structure.
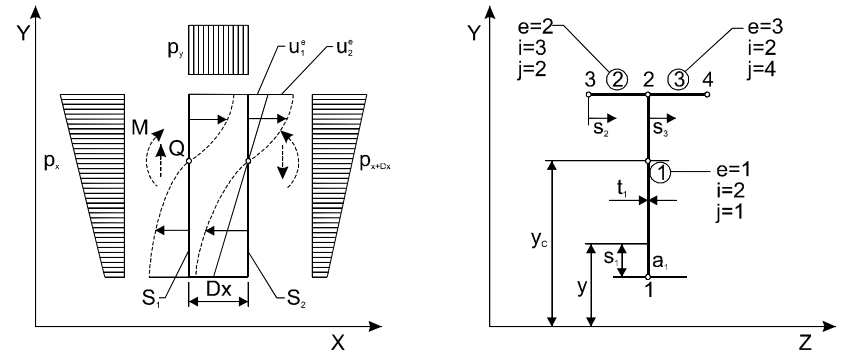

Figure 3. Cut-out from the longitudinal structural member considered as the transverse strip $\left(\mathrm{S}_{1}-\mathrm{S}_{2}\right)$ with external loading $p$ and warping fields $u$.

Primary displacement field (following classical beam theory) is defined by displacements and rota- 
tions of the cross section as a whole. Secondary displacement field $\mathbf{u}_{2}(\mathrm{x}, \mathrm{y}, \mathrm{z}) \equiv \mathbf{u}(\mathrm{x}, \mathrm{y}, \mathrm{z})$ represents warping (deplanation) of the cross section. For piecewiselinear FEM idealization of the cross section, divided into $n$ elements, with shape functions $\mathbf{N}$ in element coordinate system $(\mathrm{x}, \mathrm{s})$, the warping field reads:

$$
u(s)^{e}{ }_{x=x_{0}}=\mathbf{N}^{\mathrm{T}} \cdot \mathbf{u}^{e}=\left\{1-\frac{s}{l^{e}} \frac{s}{l^{e}}\right\}\left\{\begin{array}{l}
u_{i} \\
u_{j}
\end{array}\right\}
$$

Element strain and stress fields $\varepsilon$ and $\sigma$ are obtained from strain-displacement and stress-strain relations:

$$
\begin{aligned}
& \varepsilon \rightarrow \gamma_{x s}=\frac{\partial u}{\partial s}=\mathbf{B}^{T} \mathbf{u}^{e}=\left\{-\frac{1}{l^{e}} \frac{1}{l^{e}}\right\}\left\{\begin{array}{l}
u_{i} \\
u_{j}
\end{array}\right\} \\
& \sigma \rightarrow \tau_{x s}=G^{e} \gamma_{x s}=G^{e} \mathbf{B}^{T} \mathbf{u}^{e}
\end{aligned}
$$

Total potential energy of the $\Delta x$-long transverse strip, or segment, of the beam (with section divided into $n$ elements):

$$
\begin{aligned}
\Pi & =\sum_{n}\left[\int_{V^{e}} \sigma^{T} \varepsilon d V-\int_{S^{e}} p(x, s) u(s) d S\right]= \\
& =\sum_{e}\left[\int_{V^{e}} \frac{1}{2} \mathbf{B}^{T} \mathbf{B} d V-\int_{S^{e}} F(s) u(s) d S\right]= \\
& =\Delta x \sum_{e}\left[\frac{1}{2} \mathbf{u}^{e T} \mathbf{K}^{e} \mathbf{u}^{e}-\mathbf{u}^{e T} \mathbf{F}^{e}\right]
\end{aligned}
$$

where $p(x, s)=$ external loading on two cross sections $\left(\mathrm{S}_{1}\right.$ and $\left.\mathrm{S}_{2}\right)$ of the strip. The minimization of total potential energy leads to classical FEM matrix relation $\mathbf{K}_{2 \mathrm{D}} \mathbf{u}_{2 \mathrm{D}}=\mathbf{F}_{\mathbf{2 D}}$ ( shortened to $\mathbf{K u}=\mathbf{F}$ ). The element stiffness matrix for the proposed linear displacement distribution along the line element is:

$$
\mathbf{K}^{e}=\frac{G^{e} \cdot t^{e} \cdot R S^{e}}{l^{e}}\left[\begin{array}{cc}
1 & -1 \\
-1 & 1
\end{array}\right]
$$

Normal stress is varying with $x$ and $s$. In the beam segment of length $\Delta \mathrm{x}$, at a certain point of the cross section, the resultant stress is given by:

$$
\begin{aligned}
\Delta p(x, s) & \equiv \Delta \sigma(x, s)=\sigma(x+\Delta x, s)_{S 2}-\sigma(x, s)_{S 1}= \\
& =Q(x) \frac{\xi_{C}(s)}{I(x)} \Delta x=Q(x) \bar{F}(x, s) \Delta x
\end{aligned}
$$

where $\xi_{c}(s)=$ distance from the considered point to the neutral axis. Vector of the nodal loads for the element $e$ of the unsymmetrical cross section, in bending about the $Z$ axis, reads:

$$
\begin{aligned}
\mathbf{F}_{z}^{e}(x) & =Q_{y}(x) \cdot \overline{\mathbf{F}}_{z}^{e}(x)= \\
& =\frac{E^{e} Q_{y}(x) t^{e} R N^{e}}{E\left(I_{Y} I_{Z}-I_{Y Z}{ }^{2}\right)}\left[I_{Y} \cdot\left\{\begin{array}{c}
\frac{y_{i c} e^{e}}{2}+\frac{l^{2} \sin \alpha^{e}}{6} \\
\frac{y_{i c} l^{e}}{2}+\frac{l^{2} \sin \alpha^{e}}{3}
\end{array}\right\}-I_{Y Z} \cdot\left\{\begin{array}{c}
\frac{z_{i c} l^{e}}{2}+\frac{l^{e^{2}} \sin \alpha^{e}}{6} \\
\frac{z_{i c} l^{e}}{2}+\frac{l^{e^{2}} \sin \alpha^{e}}{3}
\end{array}\right\}\right]
\end{aligned}
$$

For the case of bending about the $Z$ axis the matrix relations $\mathbf{K u}=\mathbf{F}$ with $\mathbf{u}=Q(x) \cdot \overline{\mathbf{u}}$, can be converted into expressions $\mathbf{K} \overline{\mathbf{u}}=\overline{\mathbf{F}}$ for the warping due to unit load $\overline{\mathbf{F}}$. For the node warping $u_{i}(x)$, unit warping $\overline{\mathbf{u}}(x)$ must be multiplied with $Q(\mathrm{x})$.

Embedment of the presented methodology into the overall incremental-iterative procedure for the ultimate strength assessment rests on the following rational assumption. Each cross section of the hull girder has particular ratio of the vertical bending moment and the vertical shear force derived from their respective distributions for the considered loading condition. This ratio is approximated as constant during the incrementation of the hull girder curvature/moment, enabling the calculation of the respective shear force within each increment. This, in a turn, implies that additional iterative loop should be placed over the balancing loop for each incremental step, since the value of the moment used for the calculation of the shear force should be equal to the one obtained by the summation of the contributions of the balanced hull module principal structural members.

Since the instantaneous hull module neutral axis vertical position is changed during the iteration and/or incrementation, the respective equivalent hull module moment of inertia, as well as the instantaneous load vector for hull module vertical bending should be updated accordingly, in order to produce valid unitary warping distribution used for the calculation of the hull module shear stress distribution. For this purpose, 'efficiency' of the principal structural members is represented by the ratio of their Young's modulus and their instantaneous secant modulus, where instantaneous secant modulus is calculated for each principal structural member as the ratio between the stress and strain corresponding to the last valid equilibrium state.

Furthermore, unitary warping distribution calculation methodology assumes higher resolution discretization of the cross sectional model when compared to the incremental-iterative method used for the ultimate strength assessment. Therefore, principal structural members are assigned with their respective line elements (with averaged nodal values of the unitary warping displacements), and the value of the unitary warping displacement is calculated for each of them in the following manner:

- Stiffener-plate combinations are assigned with the five line elements in a case of the T-profile stiffener with the attached plating. Two for the both parts of the flange, two for the both parts of the attached plating and one for the web. If the stiffener cross section is of angle or flat bar type (bulbs are represented as equivalent T-profiles), the assigned number of line elements is either four or three respectively. Since the plating line elements are always characterized by the considerably greater value of the unitary warping dis- 
placement than the stiffener line elements, the greater value among those two is chosen as representative.

- Transversely stiffened plates are represented by one line element, since only plating is longitudinally relevant, and the average value of that line element's nodal unitary warping displacements is accepted as the representative one.

- Knuckles are assigned with two line elements, while multiple panel intersections are assigned with the number of line elements equal to the number of intersecting panels. Representative value for each of those principal structural elements is calculated as the average value of the group.

When distribution of the unitary warping displacements of the hull module is known, the shear stress distribution can be calculated using the following expression for the shear stress of the considered principal structural member:

$$
\tau^{P S M}=Q \cdot \operatorname{MAX}\left(G^{e} \frac{\bar{u}^{e}}{l^{e}}\right)^{p}
$$

where $\tau^{P S M}=$ shear stress of considered principal structural member; $Q=$ instantaneous shear force value acting on the hull module; $e$ denotes properties of the considered line element; $p$ denotes plating.

In effort to quantify the influence of the calculated shear stress on the stress obtained by the means of the valid stress-strain curves for every principal structural member, the following elliptic interaction formula (Yao et al. 2005) is used, both for compression and tension:

$$
\left(\frac{\sigma_{M}^{P S M}}{\sigma_{L}^{P S M}}\right)^{2}+\left(\frac{\tau^{P S M}}{\tau_{U}^{P S M}}\right)^{2}=1
$$

where $\tau^{P S M}=$ calculated shear stress of considered principal structural member; $\tau_{U}^{P S M}=$ ultimate shear strength of the plating in compression/tension; $\sigma_{M} P S M$ $=$ longitudinal stress modified with the influence of the shear stress; $\sigma_{L}{ }^{P S M}=$ longitudinal stress derived from valid stress-strain curves.

If the considered principal structural member is in compression, then the ultimate edge shear strength of the proprietary plating is considered according to the criteria given by (Paik 2003):

$$
\frac{\tau_{U}}{\tau_{Y}}= \begin{cases}1.324 \frac{\tau_{E}}{\tau_{Y}} & \text { for } 0<\frac{\tau_{E}}{\tau_{Y}} \leq 0.5 \\ 0.039\left(\frac{\tau_{E}}{\tau_{Y}}\right)^{3}-0.274\left(\frac{\tau_{E}}{\tau_{Y}}\right)^{2}+0.676 \frac{\tau_{E}}{\tau_{Y}}+0.388 & \text { for } 0.5<\frac{\tau_{E}}{\tau_{Y}} \leq 2.0 \\ 0.956 & \text { for } \frac{\tau_{E}}{\tau_{Y}}>2.0\end{cases}
$$

where $\tau_{Y}=$ yield stress of the material under pure shear loading according to von Mises criteria:
$\tau_{Y}=\frac{\sigma_{Y}}{\sqrt{3}}$

where $\sigma_{Y}=$ specified yield stress of the material; $\tau_{E}=$ elastic shear buckling stress of the simply supported plates:

$$
\tau_{E}=k_{\tau} \frac{\pi^{2} E}{12\left(1-v^{2}\right)}\left(\frac{t}{b}\right)^{2}
$$

where $k_{\tau}=$ buckling coefficient for the shear loading, dependant on the aspect ratio of the considered plate:

$k_{\tau} \approx\left\{\begin{array}{l}4.0\left(\frac{b}{a}\right)^{2}+5.34 \text { for } \frac{a}{b} \geq 1 \\ 5.34\left(\frac{b}{a}\right)^{2}+4.0 \text { for } \frac{a}{b}<1\end{array}\right.$

$\sigma_{M}{ }^{P S M}$ is calculated using (9):

$\sigma_{M}^{P S M}=\sigma_{L}^{P S M} \sqrt{1-\left(\frac{\tau^{P S M}}{\tau_{U}^{P S M}}\right)^{2}}=\sigma_{L}^{P S M} K_{\tau}^{C}$

If the considered principal structural member is in tension, then the ultimate edge shear strength of the proprietary plating is considered according to the von Mises criteria, which gives $\tau_{U}=\tau_{Y}$. In this case $\sigma_{M}{ }^{P S M}$ is calculated according to the following expression:

$\sigma_{M}^{P S M}=\sigma_{L}^{P S M} \sqrt{1-3\left(\frac{\tau^{P S M}}{\sigma_{Y}}\right)^{2}}=\sigma_{L}^{P S M} K_{\tau}^{T}$

$K_{\tau}^{C}$ and $K_{\tau}^{T}$ represent shear influence coefficients of the considered principal structural member in compression or tension, respectively. According to their definition given in equations (14) and (15) it can be seen that calculated shear stress value can not exceed the considered value of the ultimate shear stress, which is therefore considered as the limiting value. In this boundary case (when calculated shear stress is equal to the ultimate shear strength in compression or yield stress in tension) the shear influence coefficients will assume the value of zero, which will effectively exclude the considered principal structural member from the balancing procedure and annihilate it's contribution to the sectional vertical bending moment.

It should also be noted that if the used ratio of the hull module vertical bending moment and the shear force is small (actual limit value is dependant of the structural model layout), a large values of the shear force will be imposed throughout the incrementation sequence. This might cause a very large values of the shear stress in a considerable number of the hull module principal structural members and the balancing procedure might fail to find the equilibrium state 
of the section due to large longitudinal stress reductions induced by the influence of the shear stress.

\subsection{Deck efficiency coefficients}

Important drawback of the basic method is that it assumes linear strain distribution over the hull module height. This constrains the consideration of the shear lag effects and limits the evaluation of the hull girder longitudinal strength significantly, especially in the case of multi-deck ships with complex transverse sections, where some discontinuous longitudinal hull girder components are imposed with reduced deformation. Here, linear distribution assumed by the basic method, and given by the equation (16), can not be considered as accurate.

$\varepsilon_{e}=\Phi_{H M} y_{e}$

where $\varepsilon_{e}=$ strain imposed on considered principal structural element by hull module curvature $\Phi_{H M} ; y_{e}$ $=$ distance from the centroid of the considered principal structural element to the hull module effective horizontal neutral axis.

An approximate procedure using linear-elastic 3D FEM analysis is used for prediction of the efficiency of each principal structural element in order to obtain more accurate average strain as input for the stress-strain curves.

Stress of each principal structural element can be obtained from the rapid 3D FEM analysis of the generic coarse mesh hull girder model (Zanic et al. 2009). The ratio of this stress and the stress calculated by the means of the Bernoulli-Euler beam idealization can be interpreted as structural efficiency coefficient (Biot et al. 2006):

$$
k_{e}=\frac{\left(\sigma_{F E M}\right)_{e}}{\left(\sigma_{E B}\right)_{e}}=\frac{E_{e}\left(\varepsilon_{F E M}\right)_{e}}{E_{e}\left(\varepsilon_{E B}\right)_{e}}=\frac{\left(\varepsilon_{F E M}\right)_{e}}{\left(\varepsilon_{E B}\right)_{e}}
$$

where $\left(\varepsilon_{F E M}\right)_{e}=$ primary strain imposed on considered principal structural element obtained by the means of the 3D FEM analysis; $\left(\varepsilon_{E B}\right)_{e}=$ strain imposed on considered principal structural element obtained according to Bernoulli-Euler beam equation; $E_{e}=$ Young's module of the considered principal structural element; $\left(\sigma_{F E M}\right)_{e}=$ primary stress in considered principal structural element obtained by the means of the 3D FEM analysis; $\left(\sigma_{E B}\right)_{e}=$ stress in considered principal structural element obtained according to Bernoulli-Euler beam equation.

Strain due to instantaneous hull module curvature is multiplied by the calculated structural efficiency coefficient and this product is used as input into relevant stress-strain curves. If contribution of the decks to the hull girder strength is to be evaluated, an average structural efficiency or deck efficiency coefficient valid for every principal structural element of the particular deck can be defined as an av- erage value of the structural efficiency coefficients of all deck components. Since the total deck axial force is equal whether calculated as the sum of the forces on the deck components considering their individual structural efficiency coefficients, or by overall approach using the average structural efficiency coefficient, the definition of the deck efficiency coefficient is given by:

$$
k_{D}=\frac{\sum_{i=1}^{N}\left(A_{e} k_{e} \sigma_{e}\right)_{i}}{\sum_{i=1}^{N}\left(A_{e} \sigma_{e}\right)_{i}}
$$

where $N=$ number of primary structural elements of the deck; $A_{e}=$ area of the cross section of the considered primary structural elements of the deck; $\sigma_{e}=$ stress of the considered structural elements of the deck.

This is valid since strain imposed on each principal structural element is not contributed by the direct interaction of the adjacent elements. Due to the decoupled nature of the principal structural elements, the imposed strain is determined solely by the distance of the considered element from the horizontal neutral axis.

Since the same value of the $k_{e}$ is used throughout the curvature incrementation sequence, implementation of this modification has some obvious limitations regarding overall accuracy, but relatively simple and not so time consuming nature of the procedure enables better structural response assessment and renders this modification of the basic method as convenient for the application within the optimization based concept design loop.

\section{EXAMPLES OF APPLICATION}

\subsection{Chemical tanker}

The work on example of application presented herein was originally performed by the UZ-FMENA within the scope of the FP6 project IMPROVE (Naar at al. 2008), where longitudinal ultimate strength evaluation was performed for the hull girder of the 40000 DWT ocean-going chemical tanker (Figure 4) designed to carry large variety of different cargoes in thirty cargo tanks, as one of the three specific products considered by the project. The main particulars of the vessel are as follows (SSN 2008):
Length overall:

Length between perpendiculars:

Beam molded:

Depth to main deck:

Capacity of Duplex cargo tanks:

Service speed:
Scantling Draught:

Cargo tanks capacity (total):
$182.88 \mathrm{~m}$;

$175.25 \mathrm{~m}$;

$32.20 \mathrm{~m}$;

$15.00 \mathrm{~m}$;

$11.10 \mathrm{~m}$;

$44000 \mathrm{~m}^{3}$;

$26800 \mathrm{~m}^{3}$;

15.0 Knots. 


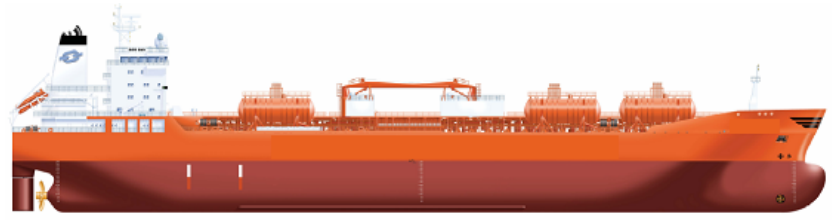

Figure 4. Chemical tanker designed by the SSN, Szczecin, Poland (SSN 2008).

Non-linear FEM analysis of the prismatic structural model of the vessel was performed (Naar et al. 2008) for two extreme loading scenarios (briefly described in section 3.1.2) and the results obtained were used in section 3.1.3 for comparison only.

\subsubsection{Structural model}

Since the structure was considered as prismatic, only the product's midship section (one-bay) model was produced and analyzed. Structural layout, main structural dimensions, as well as the stiffener scantlings used are given by the Figure 5 .

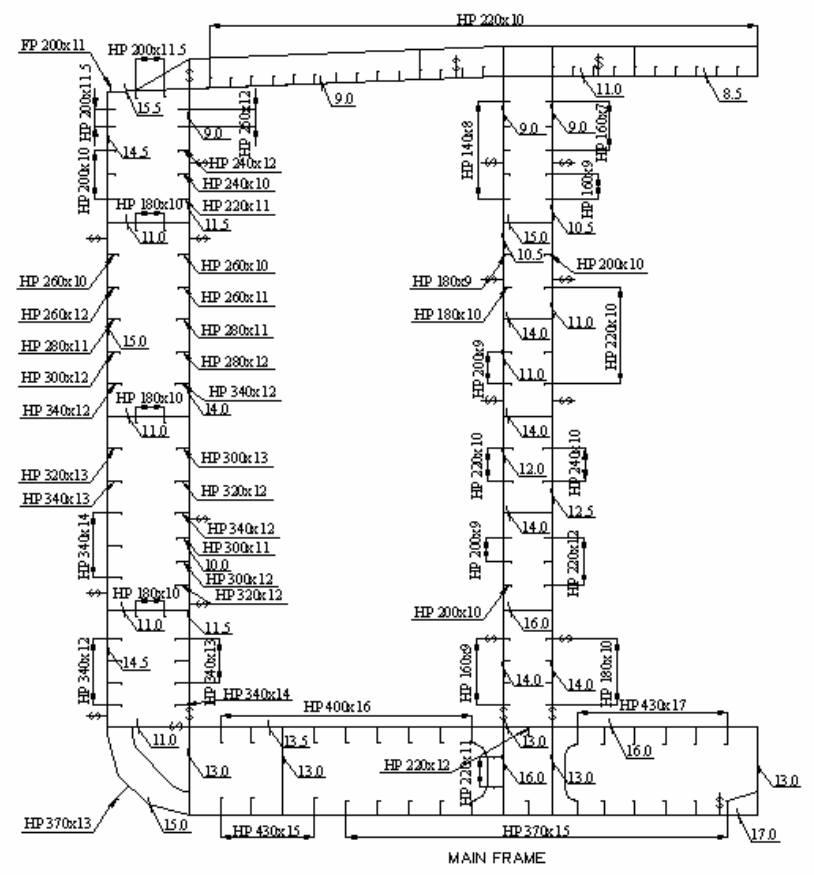

Figure 5. Midship section drawing of the analyzed structure (Naar et al. 2008).

Two different materials were used for structural elements, namely: high tensile steel (AH36) and stainless steel. Stainless steel is used only for the cargo tank plating (inner plating of the double sides and double bottom, cofferdam plating and strength deck plating), while high tensile steel is used for the rest of the structure. Relevant material properties of the materials used are specified by table 1 .

Table 1. Properties of the structural material.

\begin{tabular}{lll}
\hline Material property & High tensile steel & Stainless steel \\
\hline Youngs modulus $\left(\mathrm{N} / \mathrm{mm}^{2}\right)$ & 210000 & 210000 \\
Poisson ratio (-) & 0.3 & 0.3 \\
Yield stress $\left(\mathrm{N} / \mathrm{mm}^{2}\right)$ & 355 & 455 \\
\hline
\end{tabular}

Span of the considered one bay model is 3560 $\mathrm{mm}$, while the unsupported lengths of the transversely stiffened and cross stiffened panels of the cofferdam are: $890 \mathrm{~mm}$ (for the panels below the height of $5100 \mathrm{~mm}$ ) and $1780 \mathrm{~mm}$ (for the panels above the height of $5100 \mathrm{~mm}$ ). One bay model of longitudinally relevant structure produced using MAESTRO modeler and used as input for the longitudinal ultimate strength assessment using OCTOPUS software is given by the Figure 6.

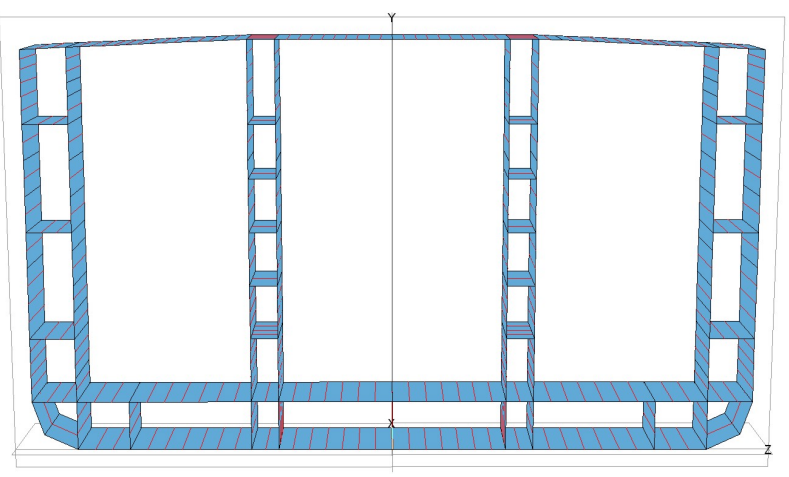

Figure 6. MAESTRO one bay structural model of the analyzed midship section.

Structural model of the chemical tanker midship section discreticised with the structural elements supported by the IACS incremental-iterative method (stiffener - plate combinations, hard corners, transversely stiffened plates) is given by the Figure 7.

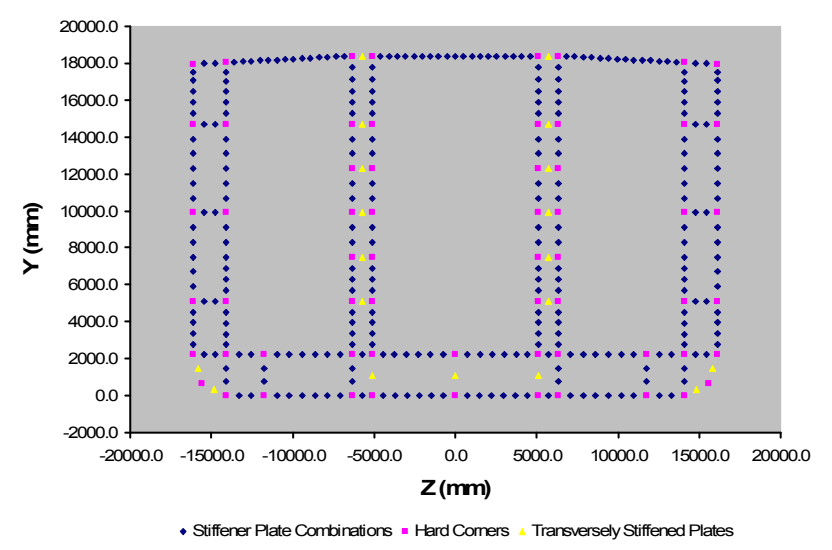

Figure 7. Longitudinal ultimate strength model of the analyzed midship section.

\subsubsection{Loading scenarios}

Two loading conditions of the vessel were identified and considered as extreme ones (Naar et al. 2008). In both cases the realistic loading distributions were determined using hydrostatic analysis. First considered loading scenario is the one giving the maximum hull girder vertical bending (at corresponding frame station), which constitutes the usual approach for the longitudinal ultimate strength assessment. Relevant distributions for this loading scenario are given by Figure 8. 


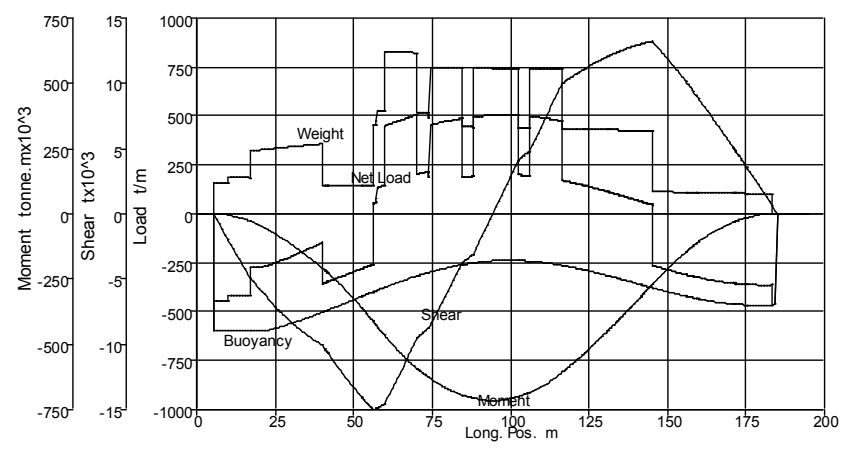

Figure 8. Loading distributions for the first extreme loading scenario (Naar et al. 2008).

The second extreme loading scenario corresponds to the loading condition with the large value of shear force and the hull girder section where the value of the shear force to vertical bending moment ratio is relatively high and might have an impact on the collapse of the hull girder. Relevant distributions for this loading scenario are given by Figure 9 .

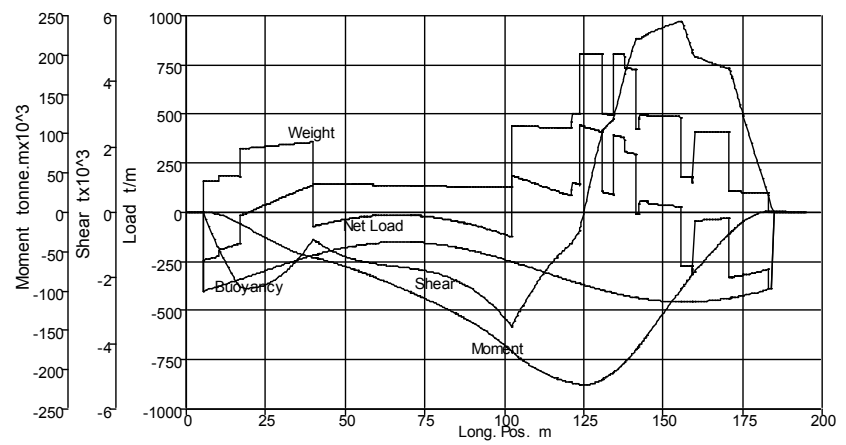

Figure 9. Loading distributions for the second extreme loading scenario (Naar et al. 2008).

\subsubsection{Results}

For the given particular example of application consideration of deck efficiency coefficients was disregarded due to the structural configuration of the analyzed hull girder (single-deck structure) and only shear stress influence was included into analysis.

Since the first loading scenario (LC1) is analyzed at the section with maximum vertical bending moment where the shear force is negligible, the moment to curvature relationship for sagging and hogging of the hull girder indicated by the Figure 10 is obtained by the means of the original (unmodified) IACS incremental-iterative method. The plot corresponding to second extreme loading scenario (LC2) is obtained with included shear stress influence, and is also given in Figure 10. Values of the ultimate vertical bending moments obtained by extended IACS incremental-iterative method (as implemented in OCTOPUS software) for both loading scenarios in sagging and hogging are given by Table 2 .
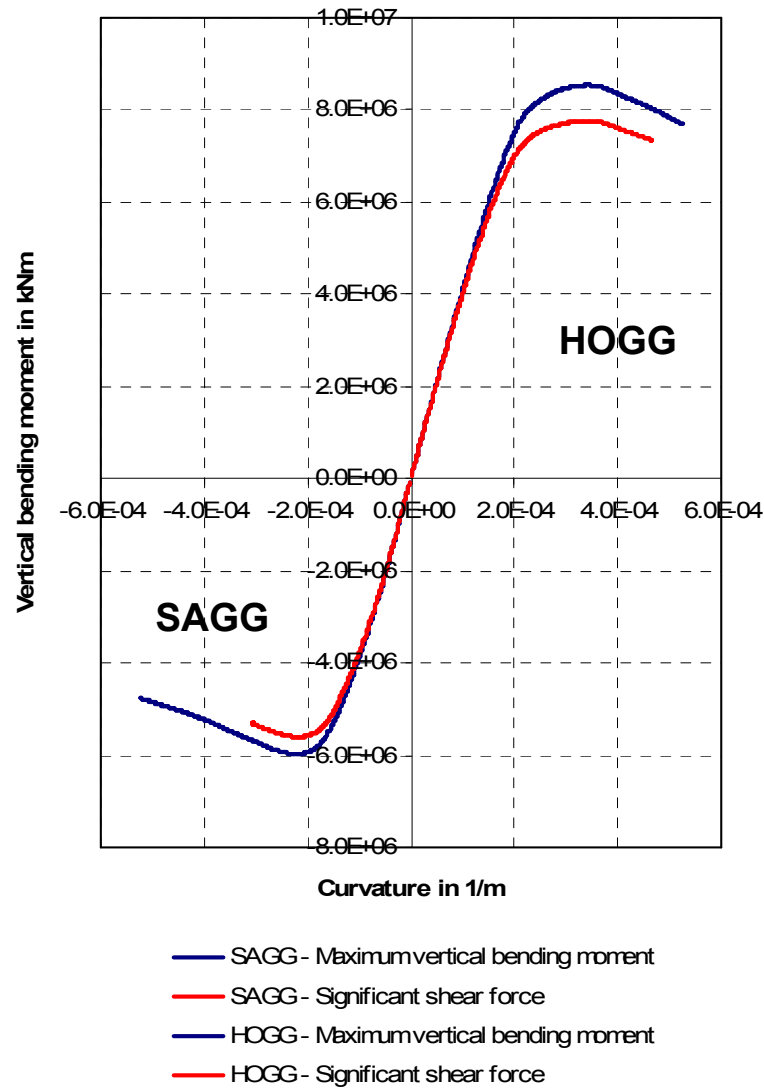

Figure 10. Hull girder vertical bending moment to curvature relationships obtained for LC1 and LC2.

Table 2. Results obtained by the extended IACS incrementaliterative method for both loading scenarios (values in $\mathrm{kNm}$ ).

\begin{tabular}{lll}
\hline & SAGG & HOGG \\
\hline LC1 $\left(\max . \mathrm{M}_{\mathrm{V}} ; \mathrm{Q}_{\mathrm{V}} \approx 0\right)$ & $-5.983 \times 10^{6}$ & $+8.538 \times 10^{6}$ \\
LC2 $\left(\max . \mathrm{Q}_{\mathrm{V}} ; \mathrm{M}_{\mathrm{V}} \neq 0\right)$ & $-5.608 \times 10^{6}$ & $+7.753 \times 10^{6}$ \\
\hline
\end{tabular}

Nonlinear FEM analysis performed showed that the analyzed hull girder structure will collapse at the section of the maximum vertical bending moment for both loading scenarios considered, rendering the comparison of the results obtained by two methods employed not valid for the LC2 (since the structural collapse occurred at the different hull girder stations). Results obtained by the NL FEM are summarized in the Table 3.

Generally, based on the results obtained, it can be argued that neglecting of the shear induced effects for hull girder transverse sections with significant shear loading leads to overly optimistic and possibly unsafe estimate of the ultimate bending capacity.

Table 3. Results obtained by the nonlinear FEM analysis for both loading scenarios considered (values in $\mathrm{kNm}$ ).

\begin{tabular}{lll}
\hline & SAGG & HOGG \\
\hline LC1 $\left(\max . \mathrm{M}_{\mathrm{V}} ; \mathrm{Q}_{\mathrm{V}} \approx 0\right)$ & $-5.830 \times 10^{6}$ & $+8.630 \times 10^{6}$ \\
LC2 $\left(\max . \mathrm{M}_{\mathrm{V}} ; \mathrm{Q}_{\mathrm{V}} \approx 0\right)$ & $-6.100 \times 10^{6}$ & $+7.420 \times 10^{6}$ \\
\hline
\end{tabular}




\subsection{Multi-deck structure}

Application of the procedure given in the section 2.3 is exemplified by the analysis of the generic structure of the simplified passenger ship, namely the 'ISSC benchmark' (ISSC 2006). Specification of the structural layout, dimensions and loading used by the various authors/contributors involved in analysis of this reference structure by various proprietary methods (including the nonlinear FEM analysis) is given by the same reference. Here, only results of the nonlinear FEM analysis will be used for comparison purposes. Figure 11 depicts the procedure of determination of the deck efficiency coefficients for this particular example of application (Andric 2007).

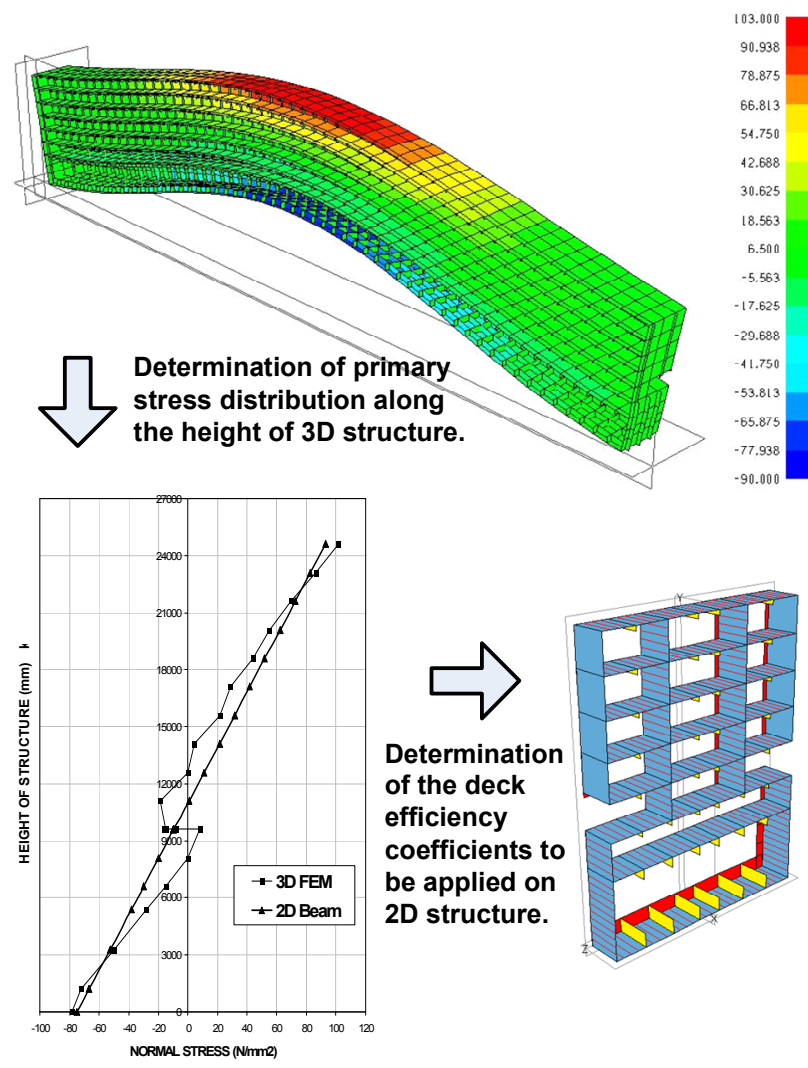

Figure 11. Procedure for determination of the deck efficiency coefficients using the prismatic ISSC benchmark model.

The results of the hull girder ultimate strength analyses performed by all three methods available (original IACS incremental-iterative, extended IACS incremental-iterative and nonlinear FEM) are presented superimposed in Figure 12, and are summarized in terms of the obtained ultimate vertical bending moments in Table 4.

Table 4. Results obtained by the three methods available for hogging and sagging loading scenarios (values in $\mathrm{kNm}$ ).

\begin{tabular}{llll}
\hline & Unmodified IACS & Extended IACS & NL 3D FEM \\
\hline HOGG & $+2.52 \times 10^{6}$ & $+2.30 \times 10^{6}$ & $+2.44 \times 10^{6}$ \\
SAGG & $-1.95 \times 10^{6}$ & $-1.80 \times 10^{6}$ & $-1.78 \times 10^{6}$ \\
\hline
\end{tabular}

The results presented show excellent agreement with the reference results of the nonlinear FEM for the sagging case, while for the hogging case reference results are approached from the safe side.

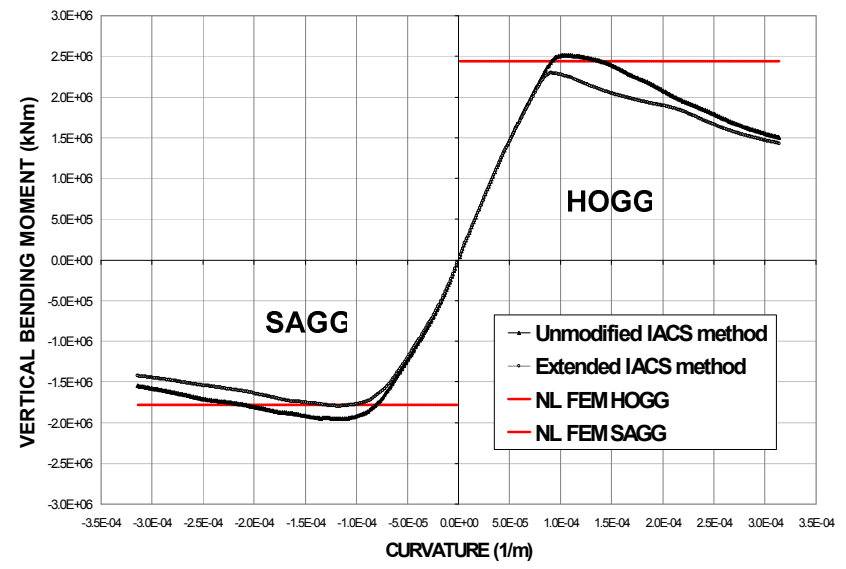

Figure 12. Resulting moment to curvature plots obtained by both unmodified and extended IACS incremental-iterative methods superimposed with the results of the nonlinear 3D FEM using the prismatic ISSC benchmark model.

Extensive validation of the implemented methodology for ultimate strength evaluation of multi-deck structures trough comparison of results obtained by nonlinear FEM and Coupled Beam (CB) method (Naar et al. 2004) analysis was performed. Multiple variants (structural openings, deletion of decks, replacement of pillars with longitudinal bulkheads, etc.) of the reference ISSC multi-deck structure were analyzed within the scope of the FP6 project IMPROVE (Naar et al. 2008).

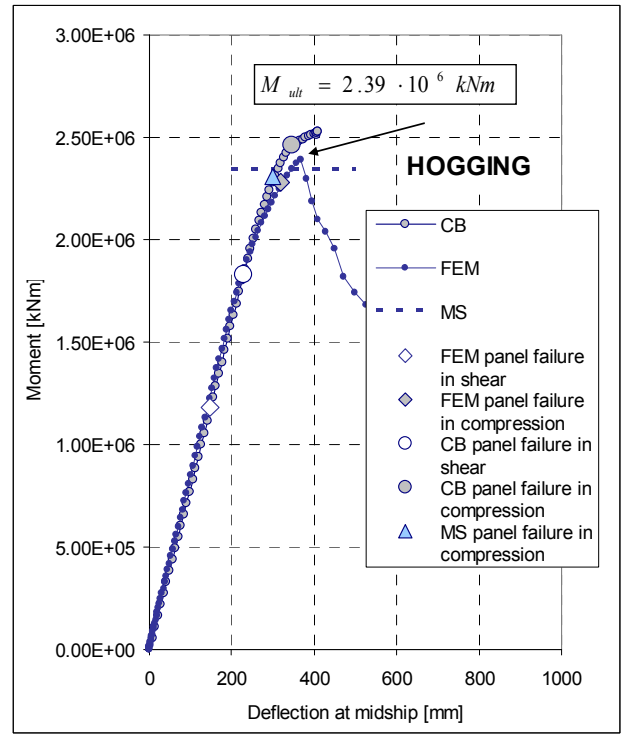

Figure 13. Moment to deflection curves at midship section for prismatic multi-deck structure with deck openings in hogging (Naar et al. 2008).

Very good agreement of the results was accomplished and reconfirmed (Figure 13). 
The value $M_{u l t}=2.39 \times 10^{6} \mathrm{kNm}$ is the ultimate moment obtained with NL FEM (Naar et al. 2008). For modified IACS method (denoted MS), the moment value is $M_{u l t}=2.34 \times 10^{6} \mathrm{kNm}$.

\section{APPLICATION IN DESIGN}

Incremental nature of the described concept for the ultimate strength assessment enables prediction of the structural collapse dynamics and establishment of the collapse sequence of the principal structural members of the hull module.

The knowledge about that sequence of the substructures (such as decks) composing a hull girder can give a very useful information regarding the weakest or critical structural areas. Collapse sequence for the second application case is presented in the Figure 14 for sagging case, as an example.

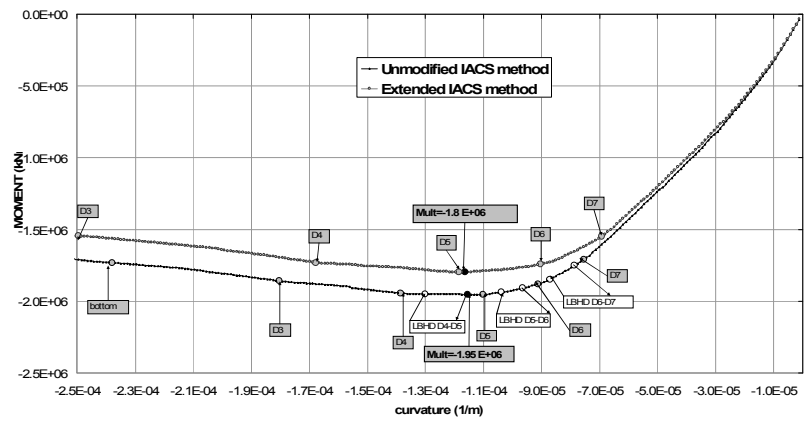

Figure 14. Collapse sequence for the sagging case.

Furthermore, identified collapse scenario can be helpful in structural optimization with the ultimate vertical bending moment as a design attribute. This enables subsequent redesign of the critical components resulting in a globally safer structure, especially if the methodology is employed within the optimization based concept design loop.

\section{CONCLUSIONS}

Considering the results of nonlinear FEM analysis for the first application case it can be concluded that the structural collapse, of the particular tanker structure considered, is not influenced by the vertical shear force induced effects. This can be generally explained by sufficient hull girder webbing (doublesides) able to effectively sustain imposed shear loading. Consequently, the structural collapse of the single-sided hull girder structure (i.e. bulk carrier) might prove more prone to shear effects considered.

Results of the hull girder ultimate strength analyses of the second application case (multi-deck) show very good agreement with the results of the nonlinear FEM analysis, especially for the sagging case.
Generally, it can be noted that the results obtained by extended IACS incremental-iterative method are in better agreement with the reference results of the nonlinear 3D FEM analysis when compared to the results of the original IACS method, enabling safer estimate of the hull girder ultimate bending capacity.

Further research has to be performed to fully understand the limitations of presented method and how it could be applied and improved for application to multi-deck structures. Detailed investigation will be performed to identify changes of the deck coefficients through design cycles and other possibilities of additional updates and improvements of the presented method.

Ultimate strength safety measure as a design objective could be very useful in multi-criteria decision making since it drives the design process towards the most rational material distribution and safer ship designs.

\section{ACKNOWLEDGMENTS}

Thanks are due to the Croatian Ministry of Science, Education and Sport for long-term support of the national project 120-1201829-1671. Considerable part of work presented in this paper was funded by the European Commission through the EU FP6 STREP project IMPROVE (Contract No. TST5-CT-2006031382). Special thanks are due to Prof. H. Naar who led MEC team from Tallinn for extensive proprietary work on loading determination, non-linear FEM modeling/analysis and the pleasant collaborative work performed on the IMPROVE project.

\section{REFERENCES}

Andric, J. 2007. Decision support methodology for concept design of the ship structures with hull-superstructure interaction. $\mathrm{PhD}$ dissertation (in Croatian), University of Zagreb, Croatia.

Biot, M. et al. 2006. Collapse Analysis of a Modern Cruise Ship Hull Girder. San Francisco: ISOPE 2006.

Hughes, O.F. 1988. Ship Structural Design - A RationallyBased Computer-Aided Optimization Approach. Jersey City: The Society of Naval Architects and Marine Engineers.

IACS 2006. Common Structural Rules for Double Hull Oil Tankers.

IACS 2008. Common Structural Rules for Bulk Carriers.

ISSC Technical Committee III.1 2006. Ultimate Strength. Proceedings of the $16^{\text {th }}$ International Ship and Offshore Structures Congress Vol 1. Southampton.

MAESTRO v8.7, Software documentation, DRS-C3 Advanced Technology Center, Stevensville, MD, USA.

Naar, H. et al. 2004. A theory of coupled beams for strength assessment of passenger ships. Marine Structure, Vol 17, Issue $8: 590-611$.

Naar, H. et al. 2008. Deliverable D3.2 - Report on Assessment of ultimate strength at the early design stage. FP6 STREP Project IMPROVE (Contract No. TST5-CT-2006-031382). 
Paik, J.K. 2003. Ultimate Limit State Design of Steel-Plated Structures. Chichester: John Wiley and Sons Ltd.

Smith, C.S. 1977. Influence of Local Compressive Failure on Ultimate Longitudinal Strength of a Ship's Hull. Tokyo: PRADS 1977.

SSN 2008. Information on Stability and Longitudinal Strength: Chemical Tanker 40000 DWT, Szczecin: IMPROVE/0423, Version 09/12/2008.

Yao, T. et al. 2004. Influence of Warping due to Vertical Shear Force on Ultimate Hull Girder Strength. LuebeckTravemuende: 9th Symposium on Practical Design of Ships and Other Floating Structures.

Zanic, V. \& Prebeg, P. 2005. Primary response assessment method for concept design of monotonous thin-walled structures. Acta Polytechnica Vol 45, No 4/2005: 96-103.

Zanic, V. et al. 2007. Decision Support Problem Formulation for Structural Concept Design of Ship Structures. Proceedings of MARSTRUCT 2007: 499-509.

Zanic, V. et al. 2009. Design Environment for Structural Design: Application to Modern Multideck Ships. Proceedings of IMechE, part M, Journal of Engineering for the Maritime Environment, Vol.223, No.M1: 105-120. 